\title{
/BIOTOOLS
}

\section{A Risk Analysis Model for Experimental Veterinary Vaccines}

\author{
A new way of analyzing risk is now available on disk
}

C Y R I L G. G A Y

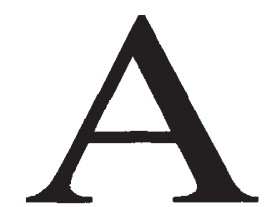

ssessing risk for any biomedical product is always a difficult task. Clearly defined standards are needed that both the regulatory agencies and the drug manufacturers agree to. For the vast majority of human drugs these standards are not yet available. But as a consequence of regulating new experimental veterinary vaccines, the U.S. Animal and Plant Health Inspection Service (APHIS, Hyattsville, MD) has developed an approach to risk assessment. Currently, this model is available on a computer diskette and allows applicants to assess risks in vaccine development as part of the application process.

The risk analysis model presented here was designed to accomplish very specific goals: (1) To ascertain whether adequate information was provided with the request; (2) to determine whether a request to ship an experimental veterinary vaccine should be approved or denied under Title 9, Code of U.S. Federal Regulations (CFR), Section 103.3; (3) to analyze whether appropriate recommendations are present to reduce or eliminate potential safety risks; (4) to prepare U.S. National Environmental Policy Act (NEPA) environmental documents (40 CFR 1508.10); and (5) to communicate the risk of the proposed request to the public.

This risk analysis model has been used to evaluate several field tests (Table 1) and they are available to the public for review (see "Acknowledgment"). APHIS hopes this risk analysis model may be extended to

TABLE 1.

Examples of risk analyses for experimental veterinary vaccines.

\begin{tabular}{|c|c|c|}
\hline Name & Type & Purpose \\
\hline Pseudo-rabies vaccine & Recombinant Vaccine & Environmental Release \\
\hline Salmonella choleraesuis vaccine & Conventional Vaccine & Environmental Release \\
\hline $\begin{array}{l}\text { (Avirulent live culture) } \\
\text { Rabies vaccine }\end{array}$ & Recombinant Vaccine & Environmental Release \\
\hline $\begin{array}{l}\text { (Vaccinia vector) } \\
\text { Toxoplasmosis vaccine } \\
\text { (Modified live protozoa) }\end{array}$ & Conventional Vaccine & Environmental Release \\
\hline $\begin{array}{l}\text { Newcastle disease-Fowl pox vaccine } \\
\text { (Fowl pox vector) }\end{array}$ & Recombinant Vaccine & Environmental Release \\
\hline $\begin{array}{l}\text { Rinderpest vaccine } \\
\text { (Vaccinia vector) }\end{array}$ & Recombinant Vaccine & Contained Study \\
\hline
\end{tabular}

Cyril G. Gay is chief staff veterinarian at the Biotechnology Section of Veterinary Biologics, Biologics, and Environmental Protection, Animal and Plant Health Inspection Service, U.S. Department of Agriculture, 6505 Belcrest Rd., Rm. 838, Federal Building, Hyattsville, MD 20782 U.S. analyzing other biotech drugs in the future.

\section{Risk assessment}

APHIS uses the standard definition of risk: The likelihood of an adverse event occurring and the consequences if that adverse event occurs. ${ }^{1}$ APHIS regards adverse events as safety hazards to animals, public health, or the environment. Consistent with accepted U.S. federal guidelines for conducting risk analysis, a safety hazard is considered to be any lack of predictability associated with an event that leads to a dangerous circumstance. ${ }^{2,3,4}$

The first step in the risk assessment for vaccines is to identify potential hazards from the characteristics of the vaccine microorganism (Table 2). To streamline this review process, APHIS supplies summary information formats (SIFs) to applicants on disk. ${ }^{5}$ Currently, four SIFs are available for vaccines: live conventional vaccines; master seed of subunit vaccines; genedeleted vaccines; recombinant vector vaccines. SIFs are designed to specify the relevant safety information to submit in support of product license applications for new veterinary vaccines. The information requested focuses on the microbiological, molecular, and biological properties of the vaccine microorganism.

In designing SIFs, APHIS recognized that the regulation of vaccines does not easily conform to check lists or rigid guidelines. Since some questions may not be relevant to a particular vaccine microorganism, a common set of scientific criteria are used to characterize the experimental vaccine. These questions are based on results from established animal safety studies. For example, determining the potential for reversion to virulence by backpassing the vaccine microorganism in the target host is an established means to assess a potential hazard. Questions in the SIFs ensure that research data are sufficient to satisfy licensing requirements by including the applicant in the risk analysis process.

\section{Release assessment} The next step is to evaluate 
the vaccine microorganism's safety characteristics within the target environment. The release assessment is a comprehensive evaluation of this potential. The model evaluates the location and characteristics of the release site; the total amount of the experimental vaccine to be used in the proposed study; the frequency and duration of exposure to the test material; and the potential for escapes into occupational, residential, or outdoor environments. Finally, the ecosystem that may be exposed to the experimental vaccine microorganism are considered.

Studies with live experimental vaccine microorganisms are classified as either contained studies or environmental releases. A contained study is an experiment physically contained in a laboratory or an animal facility. An environmental release refers to the capability for a vaccine microorganism - and its progeny-to be released into the environment. SIFs are available for each type of study.

On the basis of the hazard identification and the release assessment, the results are integrated into a risk statement that includes a likelihood rating and a consequence rating. Both of these ratings are assigned for animal safety, public health, and environmental safety. Each rating is qualified by a degree of certainty based on the data used to assign the rating. Finally, a risk rating of low, medium, or high is assigned for the purpose of decision making. Calculating all the possibilities of this system produces a total of 81 rating combinations. On the basis of these combinations, the safety risks to animals, public health, and the environment can be assessed. If they are low, the proposed study is approved. But if any of these risks are high, the request is denied. Usually requests to conduct mediumrisk studies are also denied, unless valid mitigative procedures are identified and can be implemented.

\section{Risk communication}

Risk means many different things to different people. These differences in interpretation can most often be traced to how the risk is communicated. The last phase of the risk analysis process is to effectively communicate the risks. The goal is to effectively communicate the results to industry, professional organizations, government agencies, public interest groups, and individual citizens. To assure fairness, all risk analyses are submitted to expert scientists for peer review. Further, environmental assessments are prepared and distributed. Because these groups often have differing interests, objectives, and backgrounds, this process is a complex and may be time consuming. Nevertheless, risk communication is paramount to the success of the risk analysis process.

\section{Conclusion}

The risk analysis model for experimental veterinary vaccines provides a systematic interdisciplinary approach for conducting risk assessments. This process ensures the use of scientific information in planning and decision making. The risk analysis is flexible but comprehensive. Both the likelihood of an adverse event and the consequences if that adverse event occurs are taken into consideration. Risk decisions are based on empirical data combined with information from the scientific literature.

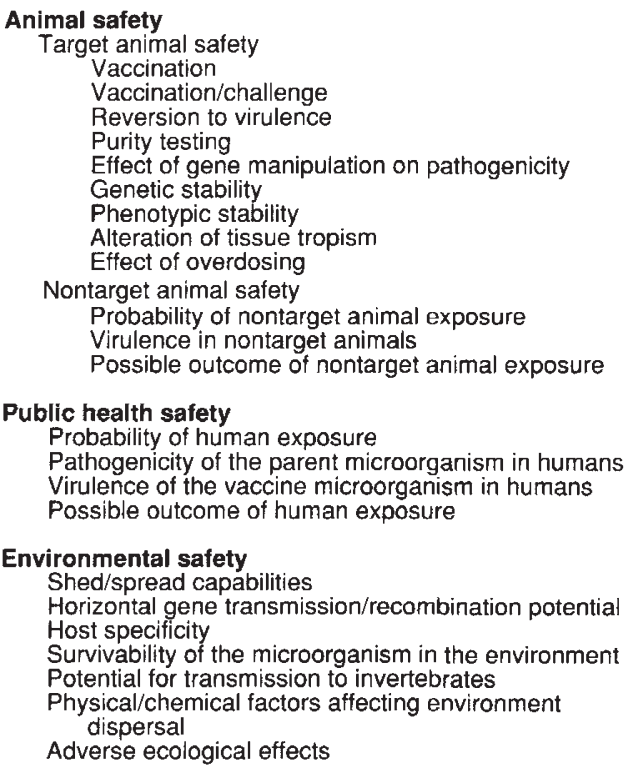

Although this risk analysis model was designed specifically to ensure the safety of experimental veterinary vaccines, the model lends itself to other risk issues. SIFs can be developed for different products or regulatory issues.

\section{Acknowledgment}

I would like to thank Richard L. Orr and H. John Roth for their advice, suggestions, and review of this article, Robert Griffin for the graphic materials, and Jeanette Greenberg for editorial assistance. Copies of risk analyses are available for public inspection by contacting Dr. Jeanette Greenberg, Veterinary Biologics, BBEP, APHIS, USDA, Room 571, Federal Building, 6505 Belcrest Road, Hyattsville, MD 20782; telephone (301) 436-5390; fax (301) 436-8669.

\section{References}

1. Ahl, A.S., Acree, J.A., Gipson, P.S., McDowell, R.M., Miller, L., and McElvaine, M.D. 1993. Standardization of nomenclature for animal health risk analysis. Rev. Sci. Tech. Off. Int. Epiz. 12 (4): 1045-1053.

2. Cohrssen, J.J., and Covello, V.T. 1989. Risk Analysis: A Guide to Principles and Methods for Analyzing Health and Environmental Risks. U.S. Council on Environmental Quality, Executive Office of the President.

3. NRC (National Research Council). 1983. Risk Assessment in the Federal Government: Managing the Process. Washington D.C.: National Academy Press.

4. NRC (National Research Council). 1992. Issues in Risk Assessment. Washington D.C.: National Academy Press. ment. Way, C.G., and Roth, H.J. 1994. Confirming the safety characteristics of recombinant vectors used in veterinary medicine: A regulatory perspective. Dev. Biol. Standard 82:89-101.

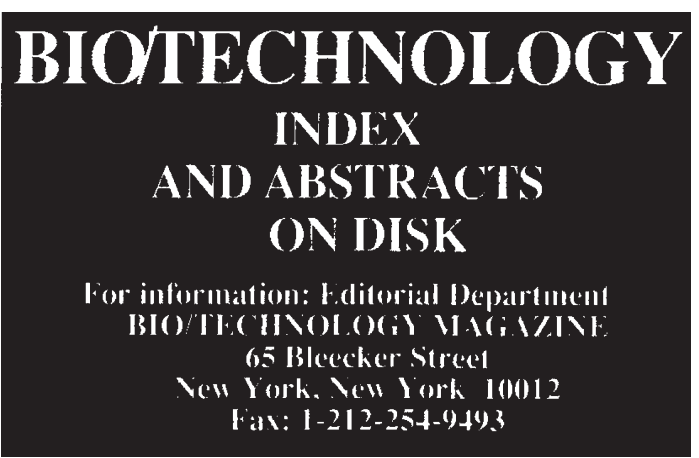

TABLE 2.

Hazard identification for veterinary vaccines. 